\title{
Identification of genes associated with the synthesis of siderophores by the Bacillus subtilis
}

\author{
T.P. Dunyashev ${ }^{1}$, G.Yu. Laptev ${ }^{1}$, E.A. Yildirim ${ }^{2 *}$, L.A. Ilina ${ }^{2}$, V.A. \\ Filippova $^{2}$, D.G. Tiurina, ${ }^{2}$ A.V. Dubrovin ${ }^{2}$, N.V. Tarlavin ${ }^{2}$ S.N. \\ Bikonya $^{2}$, E.A. Brazhnik ${ }^{2}$, V.H. Melikidy ${ }^{2}$, A.V. Platonov ${ }^{3}$
}

\author{
${ }^{1}$ Saint-Petersburg State Agrarian University, PeterburgskoyeHwy, 2, St Petersburg, 196605; ${ }^{2}$ BIOTROFLtd., \\ MalinovskayaSt, 8A, 7-N, Pushkin, StPetersburg, 196602; ${ }^{3}$ Vologda Research Center of the Russian Academy of \\ Sciences, Gorky, St, 56a, Vologda,160014, RussianFederation \\ *Corresponding author e-mail: deniz@biotrof.ru ; Ph. +7 (906) 268-92-19
}

Journal of Livestock Science (ISSN online 2277-6214) 12: 287-291

Received on 3/8/21; Accepted on 20/9/2; Published on 5/10/21

doi. 10.33259/JLivestSci.2021.287-291

\begin{abstract}
The use of biological products based on beneficial microorganisms that produce siderophores, which reduce the concentration of available to pathogensiron ions, can be successfully used in agriculture. The aim of our study was the sequencingand functional annotation of the genome of Bacillus subtilis strain from the collection of BIOTROFLtd to identify genes associated with the synthesis of siderophores. Nucleotide sequences were analyzed using a MiSeq instrument (Illumina, Inc., USA) together with a MiSeq Reagent Kit v3 (300-cycle) (Illumina, Inc., USA). Functional genome annotation was carried out using the PROKKA 1.12 (https://github.com/kbaseapps/ProkkaAnnotation http://vicbioinformatics.com/) and RAST 2.0 (https://rast.nmpdr.org) programs. The KEGG Pathway database was used to assess the pool of genes associated with antimicrobial activity and construct a metabolic map (http://www.genome.jp/kegg/). When carrying out bioinformatic processing of whole genome sequencing data of the $B$. subtilis strain, several gene clusters associated with the synthesis of siderophores were identified. Almost all genes necessary for the implementation of the process of iron binding with the participation of bacillibactin were found. A cluster of $D h b A, D h b B, D h b C$ genes associated with the production of enzymes 2,3-dihydro-2,3-dihydroxybenzoate dehydrogenase (EC 1.3.1.28), isochorismatase (EC 3.3.2.1) and isochorismates synthase (EC 5.4.4.2), which are responsible for synthesis of bacillibactin precursor was found. The cluster of genes $F e u A, F e u B, F e u C$, and $F e u D$ is associated with the synthesis of substrate-binding proteins of the iron transport system. In the genome of $B$. subtilis, genes associated with the synthesis of other siderophores were also found - enterochelin, described for E. coli and Campylobacter coli, alsomyxochelin A, first found in Angiococcusdisciformis. It is likely that $B$. subtilis bacteria can synthesize several synergistic siderophores to increase their competitiveness. In our opinion, there is a potential for using the B. subtilis strain in animal husbandry and plant growing as a basis for bacterial biopreparations - producers of siderophores, in order to reduce the pathogenic load.
\end{abstract}

Key words: Bacillus subtilis; siderophores, whole genome sequencing, probiotics, PGPR 


\section{Introduction}

Many bacteria require metal ions, in particular, ironions, which carries out the functions of electron transfer and be a cofactor in the synthesis of DNA and RNA, to ensure their vital activity (Capdevila, Edmonds, 2017). Soluble $\mathrm{Fe}^{+}$ions are usually present in the environment (water, soil, etc.) in very low concentrations (Harris, 2002). Iron homeostasis in the mammalian body is also tightly regulated; almost all iron ions in blood serum are associated with heme and proteins (ferritin, transferrin, etc.) (Andrews et al., 2003). Therefore, during the evolution microorganisms have formed specific molecular structures - siderophores - low molecular weight chelating agents that allow bacteria to assimilate iron ions in a bound state (Johnstone, Nolan, 2015). Siderophores differ in structure and chemical properties; according to the functional groups of metal chelation, they can be classified as hydroxamates, catecholates, carboxylates, and mixed types (Khan et al., 2018).

The highest efficiency of siderophore synthesis is characteristic of pathogenic microorganisms and usually correlates with their virulence level (Abaturov, Kryuchko, 2018;Koehler, 2000). Controlling the presence of iron in mammals serves as a barrier to infection (Andrews et al., 2003). It has been established that an excess of iron in microorganisms is associated with the development of a chronic course of the infectious process, since high levels of iron ions contribute to the formation of biofilms of pathogenic bacteria (Banin et al., 2005). There is evidence that the use of probiotic preparations based on beneficial microorganisms that produce siderophores, which reduce the concentration of iron ions available to pathogens, can have a positive effect on the health of animals and birds (Balakrishna, Arun Kumar, 2012).

Siderophore producers are also used in plant growing (De Serrano Luis, 2017). Due to the production of siderophores and successful competition for iron ions present in the soil, PGPR -rhizobacteria can inhibit phytopathogenic microorganisms (Rout et al., 2013). On the other hand, plant endo- and epiphytes synthesizing siderophores can contribute to improving the absorption of iron from the soil by plants, and also activate the production of the growth hormone indole-3-acetic acid (Rout et al., 2013).It is known that microbial siderophores provide plants with Fe nutrition to enhance their growth when the bioavailability of Fe is low (Crowley, 2006).

Bacillus subtilis is an aerobic gram-positive bacterium that is widely used for the production of heterologous proteins ( $\mathrm{Su}$ et al., 2020). It secretes many enzymes that break down various substrates, allowing bacteria to survive in the different environment. This species and some of its close relatives have excellent protein secreting properties, making them important hosts for the production of medicinal proteins and industrial enzymes.

Nevertheless, the pathways for biosynthesis of drugs based on siderophores are laborious, and the yield of the aimed product is low. Therefore, the use of bacterial biological products based on producers of siderophores, as natural biocontrol agents in animal husbandry and crop production, can be a very promising direction (Havenaar $\mathrm{R}$ et al., 1992;Gatesoupe FJ, 1999).

The aim of the present research was the sequencing and functional annotation of the Bacillus subtilis strain genome in order to identify genes associated with the synthesis of siderophores that are important for use in agriculture as a probiotic preparation in animal husbandry and crop production.

\section{Materials and Methods}

The material for the study was the B. subtilis strain from the collection of BIOTROF Ltd (Saint-Petersburg, Russia), which has a significant biocontrol activity. The research work was carried out in the molecular-genetic laboratory of BIOTROF Ltd. DNA isolation was carried out by standard methods using the Genomic DNA Purification Kit (Fermentas, Inc., Lithuania) according to the attached instructions. A DNA library for whole genome sequencing was prepared using the Nextera XT kit (Illumina, Inc., USA). Nucleotide sequences were determined using a MiSeq instrument (Illumina, Inc., USA) together with a MiSeq Reagent Kit v3 (300-cycle) (Illumina, Inc., USA). Invalid sequences and adapters were removed using the Trimmomatic-0.38 program (https://www.osc.edu/book/ex-port/html/4385) (Bolger et al., 2014). Filtered in length from 50 to 150 bp pairterminal sequences were assembled de novo using the SPAdes-3.11.1 genomic assembler (http://cab.spbu.ru/software/spades/). Functional genome annotation was carried out using the PROKKA 1.12 (https://github.com/kbaseapps/ProkkaAnnotation http://vicbioinformatics.com/) and $\quad$ RAST 2.0 (https://rast.nmpdr.org) programs (Aziz, 2008). The KEGG Pathway database (http://www.genome.jp/kegg/) was used to assess the pool of genes associated with antimicrobial activity and construct a metabolic map (Kanehisa et al., 2012).

\section{Results}

During bioinformatics processing of whole genome sequencing data of B. subtilis strain, several gene clusters associated with the synthesis of siderophores, such as bacillibactin, mixochelin and enterochelin (enterobactin, enterobacterin), were identified.

Table 1 shows the genes found in the genome of the studied B. subtilis strain, necessary for the transport of iron, as well as the biosynthesis of bacillibactin. For example, a cluster of $D h b A, D h b B, D h b C$ genes associated with the production of enzymes 2,3-dihydro-2,3-dihydroxybenzoate dehydrogenase (EC 1.3.1.28), isochorismatase (EC 3.3.2.1) and isochorismates synthase (EC 5.4.4.2). The genes for the assembly of bacillibactinDhbE, $D h b F$ and a cluster of genes of the iron transport system: FeuA, FeuB, FeuC, and FeuD were also found. 
Table 1. Genes and enzymes of the B. subtilis bacterial strain associated with the synthesis of bacillibactin (using the RAST database (https://rast.nmpdr.org)

\begin{tabular}{|c|l|l|}
\hline$№$ & Genename & \multicolumn{1}{c|}{ Enzymesassociatedwithgenes } \\
\hline 1 & DhbA & 2,3-dihydro-2,3-dihydroxybenzoate dehydrogenase (EC 1.3.1.28) \\
\hline 2 & DhbB & Isochorismatase (EC 3.3.2.1) \\
\hline 3 & DhbC & Isochorismatessynthase (EC 5.4.4.2) \\
\hline 4 & $D h b E$ & 2,3-dihydroxybenzoate-AMP ligase (EC 2.7.7.58) \\
\hline 5 & DhbF & Component F of bacillibactin synthetase (EC 2.7.7.) \\
\hline 6 & YuiI & Trilactonehydrolase \\
\hline 7 & FeuAa & Regulatory component: Aa iron transport system (substrate-binding protein) \\
\hline 8 & FeuA & Iron transport system A (substrate-binding protein) \\
\hline 9 & FeuB & Iron transport system B (substrate-binding protein) \\
\hline 10 & FeuC & Iron transport system C (substrate-binding protein) \\
\hline 11 & FeuD & Iron transport system D (substrate-binding protein) \\
\hline
\end{tabular}

Figure 1 shows metabolic synthesis maps (according to the KEGG Pathway database (http://www.genome.jp/kegg/)) of some siderophores, in particular, bacillibactin, in the studied B. subtilis strain. According to the obtained results (Fig. 1), the precursors for the biosynthesis of siderophores in B. subtilis are formed from the pool of amino acids in a simple way. In the first step, the resulting chorismate is isomerized to isochorismate by the enzyme chorismate-hydroxymutase (EC 5.4.4.2). Further, isochorismatase (EC 3.3.2.1) catalyzes the chemical reaction of the conversion of isochorismate to pyruvate and 2,3-dihydroxy-2,3dihydroxybenzoate, which is a precursor in the biosynthesis of these siderophores. Then the enzyme complex enterobacterin synthase ( $\mathrm{N}$ - (2,3-dihydroxybenzoyl) -serine synthetase) (EC 6.3.2.14) catalyzes the conversion of three molecules of 2,3-dihydroxybenzoate and L-serine into siderophore enterochelin. This complex is formed with the participation of the EntB and EntE gene. The EntE protein catalyzes the ATP-dependent condensation of 2,3dihydroxybenzoate and the EntB protein to form the covalently arylated form of EntB. The $M x c E$ and $M x c F$ proteins, in turn, participate in the assembly of the myxochelinsiderophore from lysine and 2,3-dihydroxybenzoic acid with the participation of ATP and NAD $(\mathrm{P}) \mathrm{H}$.

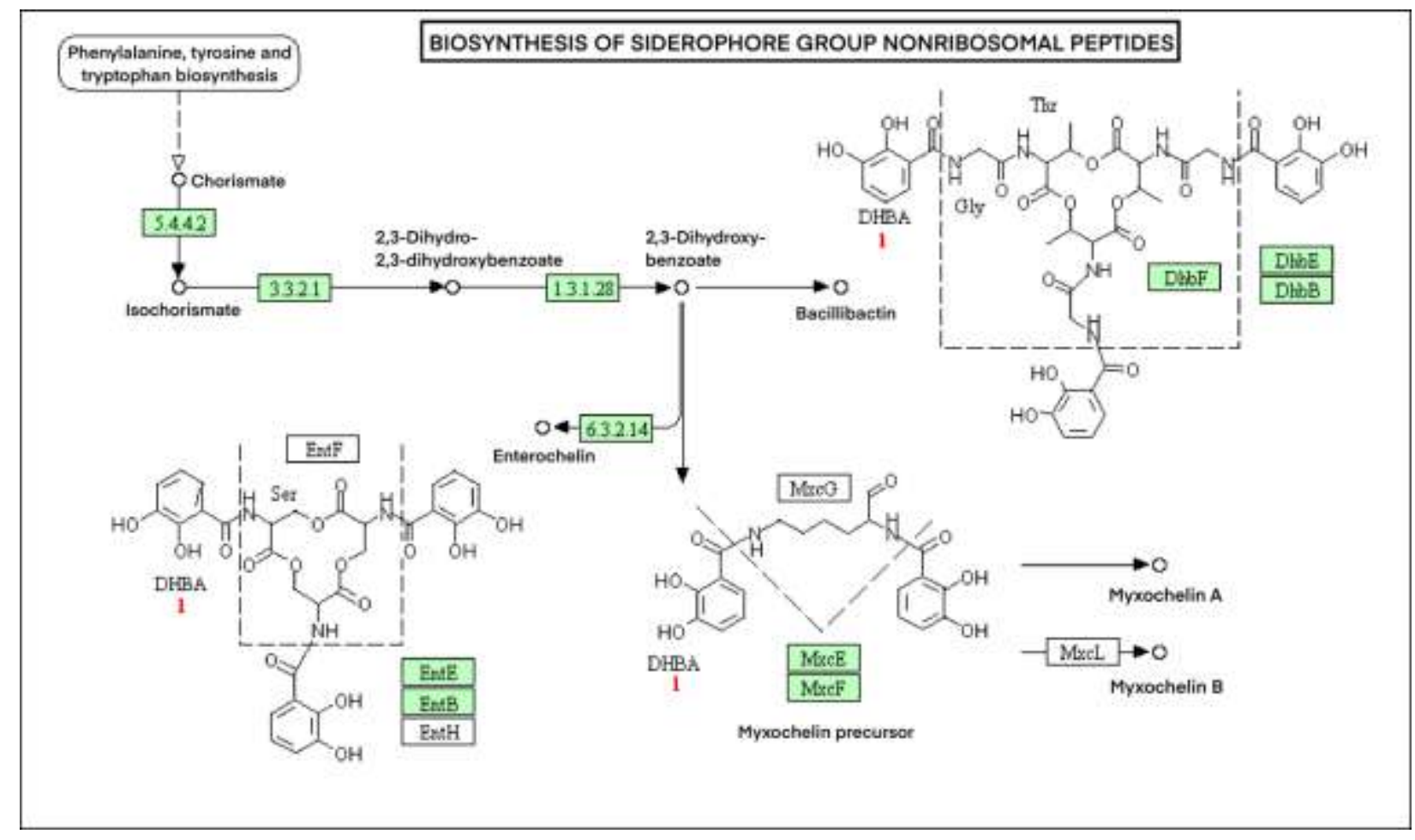

Fig. 1. Metabolic pathways leading to the formation of siderophores and their precursors in the B. subtilis bacterial strain (according to the KEGG Pathway database (http://www.genome.jp/kegg/). The rectangles indicate the proteins encoded by genes (the green ones indicate the proteins found in the B. subtilis strain), the circles indicate the various chemical compounds, the arrows indicate the molecular interactions between substances (continuous arrows indicate direct interaction, dashed arrows indicate indirect interaction) 


\section{Discussion}

The results of our study showed that in the genome of the B. subtilis strain, almost all genes necessary for the process of iron binding with the participation a bacillibactin siderophore were found. Siderophorebacillibactin is a catechol trilactone secreted by various types of bacilli during periods of reduced concentration of iron ions in the medium (Hottaet al., 2010). A quantitative assessment of intracellular and extracellular bacillibactin in the Bacillus subtilis strain revealed a fourfold increase in the concentration of this extracellular siderophore in an environment with a low content of iron ions compared to its increased content (Miethkeet al, 2006). The identified cluster of genes $D h b A, D h b B, D h b C$ is associated with the synthesis of enzymes that are responsible for the synthesis of the bacillibactin precursor. Other discovered genes - DhbE, DhbF - are involved in the assembly of bacillibactin. The cluster of genes $F e u A, F e u B, F e u C$, and $F e u D$ is associated with the synthesis of substrate-binding proteins of the iron transport system (Heinrichs et al., 2004). Miethke et al. (Miethkeet al, 2006) showed that the feuABC gene locus, encoding $\mathrm{ABC}$ transporters, including the periplasmic binding protein $\mathrm{FeuA}$ and membrane permeases $\mathrm{FeuB}$ and $F e u C$, was of decisive importance for maintaining cell growth of B. subtilis at a low concentration of iron ions in the nutrient medium. The YuiI gene is associated with the process of iron release from bacillibacterial chelate in the cytosol (Miethke et al,, 2008).

Interestingly, genes associated with the synthesis of other siderophores - enterochelin (enterobactin), described for E. coli(Langman et al., 1972) and Campylobacter coli (Richardson, Park, 1995), and alsomyxochelin A, first described forAngiococcusdisciformis (Myxobacterales) (Kunzeet al., 1989) were found (Fig. 1). Genes associated with the synthesis of secondary metabolites, including siderophores, are most prone to horizontal transfer (Ziemertet al., 2014). The identification in the genome of B. subtilis of genes associated with the synthesis of enterochelin and myxochelin A, previously found in unrelated bacteria, is a clear demonstration of evolutionary mechanisms action in relation to secondary metabolites. The persistence of these genes and even entire clusters in the genome indicates a high degree of their functionality, since their preservation is associated with an increase in genome size and replication costs. In the absence of their functionality, selection pressure would lead to the deletion of "excess genes". Probably, B. subtilis bacteria can synthesize several siderophores, acting in synergism, to increase their competitiveness, which was previously shown for other microorganisms as well (Barona-Gómez et al., 2006). In $B$. cereus, transporter proteins specific for bacillibactin and enterobactin (FeuA, FatB, FpuA, YfiY, etc.) were identified (Zawadzka et al., 2009).

This is a very important scientific and practical result, since bacterial siderophores are of great interest for their use in plant growing and animal husbandry. Previously, it was shown that treatment of wheat plants with some species of the genus Bacillus producing siderophores had a positive effect on an increase in the mass of roots and aerial parts (Ravari, Heidarzadeh, 2014). Research by Yu et al. (Yu et al., 2011) demonstrated that treatment of pepper plants with a siderophore-producing bacterial strain Bacillus subtilis suppressed fusarium wilt and stimulated plant growth. Bacillibactin produced by Bacillus subtilis AH18 was considered as a substance with the potential to be used in agriculture as a biocontrol agent (Woo, Kim, 2008).

\section{Conclusion}

Almost all genes of the B. subtilis strain necessary for the implementation of the process of iron binding with the participation of bacillibactin were found. The cluster of genes $D h b A, D h b B, D h b C$ associated with the production of enzymes which are responsible for the synthesis of the bacillibactin precursor andthe cluster of genes $F e u A, F e u B, F e u C$, and $F e u D$ is associated with the synthesis of substrate-binding proteins of the iron transport systemwas revealed.

\section{Acknowledgement}

The study was supported by the Russian Foundation for Basic Research (RFBR) grant No. 19-316-90041 "Whole-genome sequencing of bacilli strains isolated from the ruminal contents of various ruminants"

\section{References}

1) Abaturov AE, Kryuchko TA, 2018. Medicinal limitation of the availability of iron ions for pathogenic bacteria (original in Russian). Zdorov'erebenka, 13(4):416-425.

2) Andrews SC, Robinson AK, Rodriguez-Quinones F, 2003. Bacterial iron homeostasis. FEMS Microbiology Reviews, 27(2-3):215-237.

3) Aziz RK, 2008. The RAST server: rapid annotations using subsystems technology. BMC Genomics, 9(1):75.

4) Balakrishna A, Arun Kumar N, 2012. Preliminary Studies on Siderophore Production and Probiotic Effect of Bacteria Associated with the Guppy. Asian Fisheries Science, 25:193-205.

5) Banin E, Vasil ML, Greenberg EP, 2005. Iron and Pseudomonas aeruginosa biofilm formation. Proceedings of the National Academy of Sciences of the United States of America, 102(31):11076-11081.

6) Barona-Gómez F, Lautru S, Francou F-X, Leblond P, Pernodet J-L, Challis GL, 2006. Multiple biosynthetic and uptake systems mediate siderophore-dependent iron acquisition in Streptomyces coelicolor A3(2) and Streptomyces ambofaciens ATCC 23877. Microbiology, 152(11): 3355-3366.

7) Bolger AM, Lohse M, Usadel B, 2014. Trimmomatic: a flexible trimmer for Illumina sequence data. Bioinformatics, 30(15): 2114-2120. 
8) Capdevila DA, Edmonds KA, 2017.Giedroc D.P. Metallochaperones and metalloregulation in bacteria. Essays in Biochemistry, 61(2):177-200.

9) Crowley DA. 2006. Microbial siderophores in the plant rhizosphere. In: Iron Nutrition in Plants and Rhizospheric Microorganisms. (ed. LL Barton, J Abadía) Netherlands: Springer. - P. 169-189.

10) De Serrano Luis O, 2017. Biotechnology of siderophores in high-impact scientific fields. Biomolecular Concepts, 8(3-4):169-178.

11) Gatesoupe FJ, 1999. The use of probiotics in aquaculture. Aquaculture, 180 (1-2):147-165.

12) Harris WR, 2002. Molecular and Cellular Iron Transport - New York.

13) Havenaar R, Ten Brink B, Huis in't VeldJHJ, 1992. Selection of strains for probiotic use. In: Probiotics the scientific basis. (ed. R. Fuller).Chapman and Hall London. - P. 209-229.

14) Heinrichs DE, Rahn A, Dale SE, Sebulsky MT, 2004. Iron Transport in Bacteria: Molecular Genetics, Biochemistry, Bacterial Pathogenesis and Ecology. Washington, DC: American Society for Microbiology.

15) Hotta K, Kim CY, Fox DT, Koppisch AT, 2010. Siderophore-mediated iron acquisition in Bacillus anthracis and related strains. Microbiology (Reading), 156(7):1918-1925.

16) Johnstone TC, Nolan EM, 2015. Beyond iron: non-classical biological functions of bacterial siderophores. Dalton Transactions, 44(14):6320-6339.

17) Khan A, Singh P, Srivastava A, 2018. Synthesis, nature and utility of universal iron chelator - siderophore: a review.Microbiological Research, 212-213:103-111.

18) Koehler TM, 2000. Gram-Positive Pathogens. Washington, DC: American Society for Microbiology.

19) Rout ME, Chrzanowski T.H., Westlie T.K., DeLuca T.H., Callaway R.M., Holben W.E, 2013. Bacterial endophytes enhance competition by invasive plants. American Journal of Botany, 100(9):1726-1737.

20) Kanehisa M, Goto S, Sato Y, Furumichi M, 2012. Tanabe M. KEGG for integration and interpretation of largescale molecular datasets. Nucleic Acids Research, 40(D1): D109-D114.

21) KunzeB, Bedorf N, Kohl W, Höfle G, Reichenbach H, 1989. Myxochelin A, a new iron-chelating compound from Angiococcusdisciformis (Myxobacterales). Production, isolation, physico-chemical and biological properties. The Journal of Antibiotics, 42(1):14-17.

22) Langman L, Young IG, Frost GE, Rosenberg H, Gibson F, 1972. Enterochelin system of iron transport in Escherichia coli: mutations affecting ferric-enterochelin esterase. Journal of bacteriology, 112(3):11421149.

23) Miethke M, Klotz O, Linne U, May JJ, Beckering CL, Marahiel MA, 2006. Ferri-bacillibactin uptake and hydrolysis in Bacillus subtilis. Molecular Microbiology, 61(6):1413-1427.

24) Miethke M, Schmidt S, Marahiel MA, 2008. The major facilitator superfamily-type transporter YmfE and the multidrugefflux activator Mta mediate bacillibactin secretion in Bacillus subtilis. Journal of Bacteriology, 190(15):5143-5152.

25) Ravari SB, Heidarzadeh N, 2014. Isolation and characterization of rhizosphere auxin producing Bacilli and evaluation of their potency on wheat growth improvement. Archives of Agronomy and Soil Science, 60: 895-905.

26) Richardson PT, Park SF, 1995. Enterochelin acquisition in Campylobacter coli: characterization of components of a binding-protein-dependent transport system. Microbiology, 141(12):3181-3191.

27) Woo SM, Kim SD, 2008. Structural identification of siderophore (AH18) from Bacillus subtilis AH18, a biocontrol agent of Phytophthora blight disease in red-pepper. Korean Journal of Microbiology and Biotechnology, 36(4):326-335.

28) Su Y, Liu C, Fang H, Zhang D, 2020. Bacillus subtilis: a universal cell factory for industry, agriculture, biomaterials and medicine. Microbialcellfactories, 19(1): 173.

29) Yu XM, Ai CX, Xin L, Zhou GF, 2011. The siderophore-producing bacterium, Bacillus subtilis CAS15, has a biocontrol effect on Fusarium wilt and promotes the growth of pepper. European Journal of Soil Biology, 47(2): 138-145

30) Zawadzka AM, Abergel RJ, Nichiporuk R, Andersen UN, Raymond KN, 2009. Siderophore-mediated iron acquisition systems in Bacillus cereus: Identification of receptors for anthrax virulence-associated petrobactin. Biochemistry, 48(16), 3645-3657.

31) Ziemert N, Lechner A, Wietz M, Millán-Aguiñaga N, Chavarria KL, Jensen PR, 2014. Diversity and evolution of secondary metabolism in the marine actinomycete genus Salinispora. Proceedings of the National Academy of Sciences of the United States of America, 111: E1130-E1139. 